\title{
Fatores maternos relacionados à alta frequência de consumo de sacarose por crianças acompanhadas por um programa de atenção odontológica materno-infantil nos dois primeiros anos de vida
}

\author{
Maternal factors related to the high frequency of sucrose intake by \\ children followed-up by a maternal and child dental care program in \\ the first two years of life
}

Andréia Drawanz Hartwig*

Ana Regina Romano*

Fernanda Geraldo Pappen ${ }^{* *}$

Marina Sousa Azevedo ${ }^{* * *}$

\section{Resumo}

Objetivo: avaliar se os hábitos alimentares maternos relatados durante a gestação influenciam a ingestão de sacarose das crianças nos primeiros dois anos de vida. Sujeitos e método: participaram da pesquisa 204 díades mãe-filho acompanhadas por um programa de atenção odontológica materno-infantil em uma cidade no sul do Brasil. As variáveis independentes foram obtidas por meio dos prontuários odontológicos disponibilizados pelo programa. Os hábitos alimentares maternos foram coletados durante a gestação e a ingestão diária de sacarose pelas crianças foi baseada em um questionário alimentar de frequência, dicotomizado em $<4$ vezes/ dia e $\geq 4$ vezes/dia. A análise multivariada foi realizada com modelos de regressão de Poisson, com variância robusta para estimar a razão de prevalência e os intervalos de confiança de 95\%. Resultados: a prevalência do consumo de sacarose nos primeiros dois anos de vida, com uma elevada ingestão de sacarose ( $\geq 4$ vezes/dia), foi detectada em 90,69\% das crianças. Após o ajuste, o desfecho (alta ingestão de sacarose) foi 10\% maior para as crianças cujas mães relataram beber bebida açucarada para satisfazer sua sede durante a gestação [RP = 1,10 (IC95\%: 1,02-1,18)]. Conclusões: a maioria das crianças apresentou alta ingestão de sacarose em idade precoce. A triagem de mulheres grávidas para consumo de bebidas açucaradas pode ser uma maneira possível de reconhecer crianças em risco futuro de alta ingestão de sacarose na primeira infância.

Palavras-chave: Gestantes. Pré-escolares. Sacarose. Sacarose na dieta.

\section{Introdução}

A relação entre o alto consumo de alimentos açucarados e uma dieta pouco saudável, ganho de peso e aumento do risco de doenças não transmissíveis é uma preocupação para um grande número de países ${ }^{1}$. A doença crônica mais comum em crianças em todo o mundo é a cárie dentária, e a frequência de ingestão de sacarose é um elemento crucial em sua etiologia ${ }^{1}$. Dado que o consumo excessivo de sacarose pode ser um fator de risco para uma série de doenças crônicas, a Organização Mundial da Saúde (OMS) publicou recentemente uma recomendação sustentada pela Abordagem do Fator de Risco Comum de doenças, para melhorar a saúde bucal e geral de crianças e adultos de maneira mais eficiente e efetiva ${ }^{2}$.

Alguns estudos da literatura mostram que a alta ingestão de açúcar reflete uma preferência pelo sabor doce entre a maioria das crianças. No entanto, os mecanismos exatos que afetam o desejo de uma criança por doces não estão bem estabele$\operatorname{cidos}^{3}$. Ainda, pesquisas mostram que a variação individual no gosto e nas preferências alimentares pode ser influenciada geneticamente ${ }^{4}$. Os autores explicam que esse mecanismo ocorre devido à existência de algum marcador genético para o sabor que pode influenciar as preferências alimentares ${ }^{3,5,6}$. 
No entanto, alguns estudos também mostram que aspectos ambientais podem influenciar práticas alimentares individuais, particularmente durante a infância, em que a adoção de hábitos alimentares está intimamente associada aos exemplos dos pais $^{7}$. O principal cuidador, geralmente a mãe, desempenha o papel mais importante no desenvolvimento das preferências alimentares das crianças ${ }^{7}$. Um estudo americano mostrou que a dieta de má qualidade da mãe foi um forte indicador para uma dieta de baixa qualidade de seus filhos ${ }^{8}$. As mães podem influenciar as preferências alimentares de seus filhos por meio de suas próprias escolhas. Um estudo longitudinal, que acompanhou crianças de 2 a 8 anos de vida, mostrou que os alimentos não apreciados pelas mães tendem a não ser oferecidos aos seus filhos .

Com relação às experiências ambientais, quando uma criança não é exposta a uma alimentação saudável no início da vida, as consequências podem ser extremamente prejudiciais. As experiências alimentares no início da vida moldam e modificam as preferências por certos alimentos durante toda a vida ${ }^{10,11}$. A ingestão de sacarose possivelmente tem a mesma influência, pois foi demonstrado que o consumo de açúcar durante a infância, seja alto, moderado ou baixo, tende a se perpetuar no mesmo padrão para a adolescência e a idade adulta ${ }^{9}$. Existe uma preocupação crescente de que a ingestão de açúcares livres aumente o consumo total de energia e reduza a ingestão de alimentos que contenham calorias nutricionalmente mais adequadas. Portanto, o rastreamento de mulheres grávidas quanto a possíveis preditores de ingestão de sacarose, a fim de identificar crianças com risco futuro de ingestão elevada de sacarose, pode ser uma abordagem importante para reduzir a dieta não saudável e, consequentemente, as doenças crônicas não transmissíveis. Poucos estudos acompanharam díades mãe-filho desde a gestação para verificar se há associação entre a ingestão de sacarose pela mãe durante a gestação e pela criança. Assim, o objetivo deste estudo foi avaliar se os hábitos alimentares maternos relatados durante a gestação poderiam influenciar a ingestão de sacarose das crianças nos dois primeiros anos de vida.

\section{Sujeitos e método}

\section{Design do estudo e amostra}

Este estudo de coorte retrospectivo consistiu em uma amostra de conveniência de díades mãe-filho atendidas no Programa de Saúde Bucal Materno-Infantil da Universidade Federal de Pelotas, que oferece assistência odontológica preventiva e restauradora, avaliação de risco de cárie e educação para gestantes e seus bebês até os 3 anos de idade.
As principais características do serviço são a orientação sobre educação e prevenção na saúde bucal dos pares mãe-filho, bem como o incentivo aos pais/ cuidadores para estabelecer um acompanhamento odontológico regular para a criança e para si, a fim de promover hábitos saudáveis. Durante a discussão interativa, as gestantes recebem orientações de saúde bucal sobre hábitos de higiene, benefícios do uso de fluoreto, aconselhamento dietético e alimentar (alerta sobre os riscos de consumo frequente de sacarose e adição de açúcar em mamadeiras ou sucos naturais baseados no guia de orientação do Ministério da Saúde - Dez passos para uma alimentação saudável para crianças de até 2 anos) ${ }^{12}$. A orientação é fornecida e reforçada em cada visita. Todas as informações são registradas em prontuário específico de maneira padronizada.

A pesquisa foi aprovada pelo Comitê de Ética em Pesquisa antes do início do levantamento de dados (Documento no 214/2011, FO-UFPel, Brasil). Todas as mulheres grávidas assinaram termo de consentimento livre e informado por escrito antes da participação no estudo.

As entrevistas foram realizadas por estudantes de odontologia do último ano sob a supervisão direta de um professor orientador responsável por acompanhar cada nova entrevista. Para padronização dos entrevistadores, foi realizado um processo de treinamento em cada nova equipe de trabalho, duas vezes por ano. Os alunos receberam aproximadamente 6 horas de treinamento teórico, por meio de uma apresentação em que foram explicados protocolos para aplicação da entrevista utilizada no programa.

\section{Amostra}

Gestantes que foram assistidas pelo programa durante a gravidez e cujos filhos tiveram sua primeira consulta odontológica antes do primeiro ano de vida e que tiveram pelo menos uma consulta por ano durante os primeiros 3 anos de vida foram incluídas no estudo. O programa atendeu um total de 569 crianças, incluindo 229 díades mãe-filho. O estudo foi realizado com 204 díades mãe-filho, 25 díades (10,9\%), cujos prontuários não estavam completos, foram excluídos do estudo.

\section{Características socioeconômicas e demográficas}

Dados socioeconômicos e demográficos foram coletados, como idade materna, nível de escolaridade e renda familiar. A idade materna foi coletada em anos e categorizada em tercis. A renda familiar foi coletada em salários mínimos brasileiros e dividida em tercis. O nível de escolaridade foi dicotomizado em ensino fundamental ( $\leq 8$ anos) e ensino médio ou superior ( $>8$ anos). 


\section{Hábitos alimentares maternos}

Os dados sobre os hábitos alimentares maternos foram coletados durante o período da gestação, quando as mães estavam sendo acompanhadas pelo programa, por meio de três questões. As questões apresentavam respostas abertas sobre o consumo materno de bebida açucarada quando com sede ("O que você costuma beber quando está com sede?"). Após coleta, foram categorizadas como "sem açúcar" (quando as mães costumam consumir água ou outra bebida sem acréscimo de açúcar, como sucos naturais) e "açucarada" (quando as mães relatavam consumir bebidas doces e com adição de açúcar, como refrigerantes, sucos artificiais e sucos naturais com adição de açúcar). Para a pergunta sobre ingestão de sacarose materna (Você come doces?), as respostas foram classificadas em "não", "sim, às vezes" ( $<3$ vezes na semana) e "sim, todos os dias". A última pergunta foi sobre o consumo de açúcar durante a gravidez ("Você mudou seus hábitos alimentares quando soube que estava grávida?"). Aquelas mães que responderam que não mudaram ou mudaram por um alimento mais saudável, como comer mais vegetais ou frutas, foram categorizados em "Mesma ou menor ingestão de açúcar"; e as mães que responderam que comiam mais doces foram categorizadas em "aumento da ingestão de açúcar".

\section{Consumo de sacarose pelas crianças}

A frequência de consumo de sacarose pelas crianças foi a variável dependente. Foi construída com dados de um questionário de frequência alimentar (QFA) durante os primeiros dois anos de idade. O último QFA coletado foi utilizado na análise. A ferramenta QFA foi desenvolvida com base no consumo habitual de alimentos em crianças pré-escolares. As mães também foram questionadas sobre alimentos e bebidas contendo sacarose consumidos por seus filhos ${ }^{13}$. As questões foram relacionadas a doze itens: vegetais, frutas, água, açúcar no chá, refrigerante, açúcar no café, açúcar no leite, iogurtes, sucos adoçados artificialmente/suco de gelatina, bolo/biscoito doce, pão/biscoito, doces/pirulitos. A ingestão de sacarose foi contada quando relatado um alimento doce, uma bebida açucarada ou colher de açúcar adicionada. Em seguida, as mães foram questionadas sobre quantas vezes a criança consumiu o alimento ou a bebida. Na sequência, foi criada uma variável diária de consumo de açúcar, que foi dicotomizada em $<4$ vezes/dia (baixa/moderada ingestão de sacarose) e $\geq 4$ vezes/dia (alta ingestão de sacarose). Esse limiar foi estabelecido com base na diretriz da OMS sobre dieta, nutrição e prevenção de doenças crônicas ${ }^{1}$.

\section{Análise estatística}

Após coleta dos dados, foi realizada uma dupla digitação para tabulação, a fim de garantir a precisão da coleta. O software STATA versão 12.0 foi utilizado para as análises. As associações entre as variáveis foram testadas pelo teste do Qui-Quadrado e pelo teste exato de Fisher. Na análise multivariada, utilizou-se a regressão de Poisson com variância robusta para estimar a razão de prevalência e intervalo de confiança de $95 \%$. Todas as variáveis que não apresentaram células vazias na análise bivariada foram testadas na análise bruta. Todas as variáveis que apresentaram valor de $\mathrm{p}<0,25$ na análise bruta foram incluídas na análise multivariada ajustada. Um modelo conceitual foi desenvolvido para o ajuste de análises multivariadas. Neste modelo, as variáveis foram controladas para aqueles no mesmo nível ou em nível superior. O nível de número um é composto por variáveis socioeconômicas e demográficas, e o nível número dois abrange os hábitos alimentares maternos.

\section{Resultados}

A amostra do presente estudo foi composta por 204 díades mãe-filho. A prevalência do consumo de açúcar nos dois primeiros anos de vida foi bastante elevada. Uma alta ingestão de sacarose ( $\geq 4$ vezes/ dia) coletada pelo QFA foi detectada em $90,69 \%$ $(\mathrm{n}=185)$ das crianças. A Tabela 1 apresenta as características da amostra, a situação socioeconômica e demográfica e os hábitos alimentares dos sujeitos da pesquisa. A análise bivariada mostrou que não há associação entre as variáveis independentes e a alta frequência de ingestão de sacarose nas crianças (Tabela 2).

\begin{tabular}{|c|c|c|}
\hline Variáveis & $\mathrm{n}$ & $(\%)$ \\
\hline \multicolumn{3}{|l|}{ Escolaridade materna (anos)* } \\
\hline$<8$ & 83 & $(59.1)$ \\
\hline$\geq 8$ & 120 & $(40.9)$ \\
\hline \multicolumn{3}{|l|}{ Renda familiar (SMB) } \\
\hline $3.1-22$ & 42 & $(20.9)$ \\
\hline $1.6-3.0$ & 93 & $(45.9)$ \\
\hline $0-1.5$ & 69 & $(33.7)$ \\
\hline \multicolumn{3}{|l|}{ Idade materna (anos) } \\
\hline $14-24$ & 71 & $(35.1)$ \\
\hline $25-29$ & 61 & $(29.8)$ \\
\hline $30-42$ & 72 & $(35.1)$ \\
\hline \multicolumn{3}{|c|}{$\begin{array}{l}\text { Consumo materno de bebida açucarada quando } \\
\text { está com sede* }\end{array}$} \\
\hline Sem açúcar & 163 & $(80.3)$ \\
\hline Açucarada & 40 & $(19.7)$ \\
\hline \multicolumn{3}{|l|}{ Ingestão de sacarose materna } \\
\hline Não & 41 & $(20.1)$ \\
\hline Sim, às vezes & 87 & $(42.7)$ \\
\hline Sim, todo dia & 76 & $(37.3)$ \\
\hline \multicolumn{3}{|c|}{ Ingestão de sacarose durante a gravidez* } \\
\hline Mesmo ou menor & 183 & $(92.4)$ \\
\hline Consumo aumentado & 15 & $(7.6)$ \\
\hline
\end{tabular}

*Dados faltantes; SMB: salário mínimo brasileiro.

Fonte: autoras. 
Tabela 2 - Associação entre frequência de consumo de sacarose infantil e dados socioeconômico, demográfico e de consumo materno de açúcar em uma amostra de díades mãe-filho acompanhadas por um Programa de Saúde Bucal em Pelotas, Brasil (n=204)

\begin{tabular}{|c|c|c|c|}
\hline \multirow{2}{*}{ Variáveis } & \multicolumn{3}{|c|}{ Ingestão de sacarose $\geq 4$ vezes/dia, n (\%) } \\
\hline & Não & Sim & Valor de $p$ \\
\hline Total & 19(9.7) & $185(90.7)$ & \\
\hline Escolaridade materna (anos)* & & & 0.170 \\
\hline$<8$ & $5(6.0)$ & $78(94.0)$ & \\
\hline$\geq 8$ & $14(11.8)$ & 105(88.2) & \\
\hline Renda familiar (SMB) & & & $0.635^{*}$ \\
\hline $3.1-22$ & $5(11.9)$ & $37(88.1)$ & \\
\hline $1.6-3.0$ & $7(7.5)$ & $86(92.5)$ & \\
\hline $0-1.5$ & $7(10.1)$ & $62(89.9)$ & \\
\hline Idade materna (anos) & & & $0.277^{*}$ \\
\hline $14-24$ & $5(7.0)$ & $66(93.0)$ & \\
\hline $25-29$ & $4(6.6)$ & $57(93.4)$ & \\
\hline $30-42$ & $10(13.9)$ & $62(86.1)$ & \\
\hline Consumo materno de bebida açucarada quando está com sede* & & & $0.130^{*}$ \\
\hline Sem açúcar & $18(11.1)$ & $144(88.9)$ & \\
\hline Açucarada & $1(2.5)$ & $39(97.5)$ & \\
\hline Ingestão de sacarose materna & & & 0.750 \\
\hline Não & $5(12.0)$ & $36(87.8)$ & \\
\hline Sim, às vezes & $7(8.1)$ & $80(92.0)$ & \\
\hline Sim, todo dia & $7(9.3)$ & $68(90.7)$ & \\
\hline Ingestão de sacarose durante a gravidez* & & & $0.370^{*}$ \\
\hline Mesmo ou menor & $19(10.4)$ & 163(89.6) & \\
\hline Consumo aumentado & $0(0.0)$ & 15(100.0) & \\
\hline
\end{tabular}

* Teste exato de Fisher; * Dados faltantes; ¥ Variável com célula vazia não inserida no modelo de regressão.

Fonte: autoras.

Os resultados da análise multivariada são apresentados na Tabela 3. Após o ajuste para potenciais confundidores, a probabilidade do desfecho (alta ingestão de sacarose) foi $10 \%$ maior para crianças

cujas mães relataram consumo de bebidas açucaradas para satisfazer sua sede [razão de prevalência $(P R)=1,10($ IC 95\%: 1,02-1,18)].

Tabela 3 - Razões de prevalência (RP) brutas (c) e ajustadas (a) para variáveis independentes e consumo de sacarose pela criança de acordo com modelo conceitual adotado em uma amostra de díades mãe-filho acompanhadas por um programa de saúde bucal em Pelotas, Brasil $(n=204)$

\begin{tabular}{|c|c|c|c|c|c|c|}
\hline \multirow{2}{*}{ Variáveis } & \multicolumn{6}{|c|}{ Ingestão de sacarose $\geq 4$ vezes/dia, n (\%) } \\
\hline & $\mathrm{RP}^{\mathrm{c}}$ & $\mathrm{Cl} 95 \%$ & Valor de $\mathrm{p}$ & $\mathrm{RP}^{\mathrm{a}}$ & IC $95 \%$ & Valor de $p$ \\
\hline \multicolumn{7}{|c|}{ Nível 1: Variáveis socioeconômicas e demográficas } \\
\hline Escolaridade materna (anos)* & & & 0.250 & & - & \\
\hline$<8$ & 1.00 & & & & & \\
\hline$\geq 8$ & 1.05 & $(0.96-1.15)$ & & & & \\
\hline Renda familiar (SMB) & & & 0.870 & & - & \\
\hline $3.1-22$ & 1.00 & & & & & \\
\hline $1.6-3.0$ & 1.05 & $(0.92-1.19)$ & & & & \\
\hline $0-1.5$ & 1.02 & $(0.89-1.17)$ & & & & \\
\hline Idade materna (anos) & & & 0.310 & & - & \\
\hline $14-24$ & 1.00 & & & & & \\
\hline $25-29$ & 1.01 & $(0.90-1.13)$ & & & & \\
\hline $30-42$ & 0.94 & $(0.81-1.08)$ & & & & \\
\hline
\end{tabular}

\section{Nível 2: Hábitos de dieta maternos}

Consumo materno de bebida açucarada quando está com sede*

Sem açúcar

Açucarada

Ingestão de sacarose materna

Não

Sim, às vezes

Sim, todo dia

1.00
1.10

1.00
1.05
1.03

\begin{tabular}{|l|l|l|l|l} 
& 0.014 & & & 0.014 \\
& & 1.00 & & \\
& & 1.10 & & \\
$(1.02-1.18)$ & & & & \\
$(0.92-1.19)$ & & & & \\
\hline
\end{tabular}

IC = intervalo de confiança; - variáveis excluídas $(p>0,005)$ do primeiro nível do modelo conceitual; * variável excluída após ajuste de acordo com o segundo nível. Fonte: autoras. 


\section{Discussão}

As crianças incluídas neste estudo faziam parte de um programa de saúde bucal materno-infantil, que tem como um dos objetivos orientar as mães sobre a importância de uma alimentação saudável, em que aconselhamentos foram fornecidos para evitar introdução de açúcar nos primeiros anos de vida dos filhos ${ }^{1,12}$. No entanto, os resultados mostraram que, mesmo participando de um programa preventivo, aos 2 anos de idade, todas as crianças já haviam consumido alimentos açucarados. Os achados deste estudo concordaram com os de outras pesquisas ${ }^{14,15}$. Ainda, um estudo com pré-escolares mexicanos mostrou que as crianças participantes tiveram introdução de bebidas açucaradas antes dos 24 meses de idade, sendo que, para a maioria delas (73\%), essa introdução ocorreu antes dos 12 meses de idade ${ }^{15}$.

É importante destacar que a mudança de comportamento é uma tarefa muito difícil. Os determinantes do comportamento alimentar são multifatoriais e incluem uma complexa gama de fatores nutricionais, demográficos, sociais, culturais, ambientais e psicológicos. Entretanto, mudanças de comportamento na ingestão de determinados alimentos podem ter um impacto significativo na saúde dos indivíduos, tanto em curto quanto em longo pra$\mathrm{zo}^{16}$. Assim, a OMS tem estimulado pesquisadores a avaliar diferentes abordagens comportamentais para promover a redução da ingestão de açúcares livres, em especial a ingestão de bebidas com adição de açúcar, que é identificada como um fator de risco comportamental para o consumo excessivo de calorias, especialmente entre crianças ${ }^{2}$.

Tem sido demonstrado que as crianças com baixa ingestão de sacarose consomem alimentos mais densos em nutrientes e, portanto, têm melhor qualidade alimentar e maior ingestão de vitaminas e minerais do que as crianças com médio ou alto consumo de sacarose ${ }^{17}$. Assim, a OMS divulgou as diretrizes com recomendações para limitar o consumo de açúcar, por meio de alimentos e bebidas, para reduzir problemas de saúde pública ${ }^{18}$. Um guia recente da OMS fornece recomendações sobre a ingestão de açúcares livres para reduzir o risco de doenças não transmissíveis em crianças e adultos ${ }^{2}$. A OMS recomendou uma redução na ingestão de açúcares livres durante todo o ciclo de vida. Açúcares livres incluem sacarose adicionada a alimentos e bebidas pelo fabricante, cozinheiro ou consumidor, e os açúcares naturalmente presentes em alimentos, como mel, sucos de frutas e concentrados de suco de frutas, além de açúcares presentes em medicamentos ${ }^{2}$. Dessa forma, a variável "ingestão diária de sacarose" presente nesta pesquisa incluiu apenas açúcares livres, não levando em consideração açúcares ocultos presentes naturalmente em frutas e outros alimentos.
O desenvolvimento de preferências alimentares é bastante complexo e já foi demonstrado que começa no início da vida fetal e continua ao longo da vida. Esse desenvolvimento de preferências alimentares envolve uma interação complexa de influências ambientais, tendências biológicas e genéti$\operatorname{cas}^{3,4,7,19}$. O paladar e o olfato no recém-nascido podem correlacionar-se com os componentes dietéticos maternos nos líquidos amnióticos liberados pela ingestão de alimentos pela mãe durante a gestação ${ }^{19}$. Neste estudo de coorte retrospectivo, não podemos demonstrar que as mães que aumentaram a ingestão de açúcar durante a gestação tiveram filhos que apresentaram maior frequência de consumo de sacarose na infância, uma vez que essa variável não pôde ser inserida no modelo de regressão. No entanto, em um estudo de coorte entre crianças com 1 ano de idade, uma ingestão frequente de alimentos ricos em açúcar foi significativamente associada à alta ingestão de alimentos açucarados durante a gestaçã $0^{14}$. As crianças que experimentaram um sabor doce durante o desenvolvimento fetal podem estar predispostas a aceitar mais facilmente alimentos doces após o nascimento, indicando que as memórias são formadas a partir dessas experiências sensoriais iniciais ${ }^{20}$.

A maioria das gestantes está ciente de que a adoção de hábitos saudáveis durante a gravidez pode refletir em um melhor desenvolvimento do bebê ${ }^{21}$. O aumento do consumo de açúcar durante a gestação pode estar relacionado ao desrespeito materno pela sua saúde e pela saúde dos seus filhos. Portanto, mães que não fazem escolhas saudáveis durante a gravidez podem ser mais propensas a perpetuar esses hábitos não saudáveis após o nascimento da criança.

É importante ressaltar que a preferência por alguns alimentos também pode ser desenvolvida mais tarde, dependendo do alimento disponível ofertado para as crianças. A casa e o ambiente familiar têm grande influência no desenvolvimento de hábitos alimentares e comportamentos nas crianças ${ }^{22}$. Os pais servem como modelos, no que diz respeito ao comportamento alimentar da criança, pois são eles que determinarão quais alimentos estarão disponíveis $^{23}$. Os tipos de alimentos preferidos pelas famílias também estarão mais disponíveis para as crianças $^{22,24}$. Os resultados deste estudo mostraram que o consumo materno de bebidas açucaradas quando com sede, durante a gestação, teve uma associação positiva com a alta ingestão de sacarose pelos seus filhos. Portanto, mães que têm o hábito de beber bebidas doces durante o período gestacional podem transmitir o mesmo hábito para seus filhos, pois esse tipo de bebida estará mais disponível em casa ${ }^{25}$.

Embora as mães que relataram beber bebidas açucaradas quando com sede durante a gestação tenham apresentado crianças com alta ingestão de sacarose, o relato materno sobre a ingestão de açúcar não foi associado ao desfecho. No Brasil, como 
em outros países, uma dieta pouco saudável normalmente é introduzida pelas famílias nos primeiros anos de vida das crianças. Doces e guloseimas, culturalmente, têm conotações de amor e carinho, estando disponíveis na maioria da rotina alimentar das famílias. Entretanto, os efeitos nocivos do açúcar são amplamente divulgados na mídia e, por essa razão, são bem conhecidos pelos pais ${ }^{26}$. Ainda, as mães participantes da pesquisa receberam todas as orientações sobre dieta e hábitos alimentares saudáveis para seus filhos, estando cientes dos problemas causados pela ingestão precoce de açúcar. Assim, as respostas maternas podem ter sido influenciadas pelo viés de desejabilidade social com consequente subestimação do consumo de açúcar relatado pelas mães. Além disso, questões com respostas categorizadas estão sujeitas a outra desvantagem; segundo $\operatorname{Hurd}^{27}$ (1999), as perguntas de agrupamento levam o respondente a mudar sua resposta final de acordo com as categorias mencionadas na questão. Assim, nossa questão pode não ser sensível o suficiente para detectar a ingestão real de doces e balas.

Alguns pontos devem ser destacados neste estudo. Para a análise dos dados, foi utilizado o modelo de regressão de Poisson com variância robusta. A literatura epidemiológica tem mostrado que modelos de Poisson com variâncias ajustadas podem fornecer estimativas de ponto e intervalo corretas em estudos transversais e longitudinais com resultados binários, além de apresentar uma comunicação mais interpretável e mais fácil ${ }^{28}$. Todavia, considerando a complexa interação de influências ambientais, tendências biológicas, comportamentais e genéticas para o desenvolvimento de preferências alimentares, este estudo apresenta limitações. Primeiro, avaliamos apenas a influência ambiental no desenvolvimento das preferências alimentares das crianças. Além disso, usamos dados de registros de díades mãe-filho seguidos por um programa, não sendo essa amostra representativa de toda a população. O dispositivo de coleta de frequência alimentar usado pelo programa para coletar dados não foi validado. Posteriormente, os pares mãe-filho que faziam parte desse programa receberam orientação nutricional, assim, o comportamento alimentar das crianças pode ter sido modificado pelas instruções. Logo, nossos dados devem ser interpretados com cautela.

\section{Conclusões}

Neste estudo, a maioria das crianças apresentou alta ingestão de sacarose em idade precoce. Uma maior frequência de ingestão de sacarose foi encontrada em crianças cujas mães relataram consumir bebidas açucaradas durante a gestação. Dessa forma, a triagem de mulheres grávidas para consumo de bebidas açucaradas pode ser uma maneira possível de reconhecer crianças em risco futuro de alta ingestão de sacarose na primeira infância. Mais estudos são necessários para confirmar essa associação, preferencialmente utilizando instrumentos validados.

\section{Abstract}

Objective: to assess whether maternal eating habits reported during pregnancy influence their children's sucrose intake in the first two years of life. Subjects and method: a total of 204 mother-child dyads followed-up by a maternal and child oral care program in southern Brazil participated in the study. The independent variables were obtained from dental records provided by the program. The maternal eating habits were collected during pregnancy and the daily sucrose intake of the children was based on a frequency food questionnaire dichotomized in $<4$ times/day and $\geq 4$ times/day. The multivariate analysis was performed using Poisson regression models with robust variance to estimate the prevalence ratio and the $95 \%$ confidence intervals. Results: the prevalence of sucrose intake in the first two years of life was high. A high sucrose intake ( $\geq 4$ times/ day) was detected in $90.69 \%$ of the children. After adjustment, the outcome (high sucrose intake) was $10 \%$ higher for children whose mothers reported drinking sugar-sweetened beverages to satisfy their thirst during pregnancy [PR $=1.10$ (95\%Cl: 1.02-1.18)]. Conclusion: most of the children presented a high sucrose intake at an early age. Screening pregnant women for sugar-sweetened beverage intake may be a potential way to recognize the children at risk for future high sucrose intake in early childhood.

Keywords: Pregnant women. Preschoolers. Sucrose. Dietary sucrose.

\section{Referências}

1. Organização Mundial da Saúde. Diet, nutrition and the prevention of chronic diseases. Report of a Joint WHO. World Health Organ Tech Rep Ser 2003;916.

2. Organização Mundial da Saúde. Sugars intake for adults and children. Geneva: World Health Organization; 2015. p. 1-59.

3. Jamel HA, Sheiham A, Watt RG, Cowell CR. Sweet preference, consumption of sweet tea and dental caries; studies in urban and rural Iraqi populations. Int Dent J 1997; 47(4):2137.

4. Keller KL, Tepper BJ. Inherited taste sensitivity to 6-n-propylthiouracil in diet and body weight in children. Obes Res 2004; 12(6):904-12.

5. Anliker JA, Bartoshuk L, Ferris AM, Hooks LD. Children's food preferences and genetic sensitivity to the bitter taste of 6-n-propylthiouracil (PROP). Am J Clin Nutr 1991; 54(2):316-20.

6. Turnbull B, Matisoo-Smith E. Taste sensitivity to 6-n-propylthiouracil predicts acceptance of bitter-tasting spinach in 3-6-y-old children. Am J Clin Nutr. 2002; 76(5):1101-5.

7. Castilho AR, Mialhe FL, Barbosa T de S, Puppin-Rontani RM. Influence of family environment on children's oral health: a systematic review. J Pediatr 2013; 89(2):116-23. 
8. Lee SY, Hoerr SL, Schiffman RF. Screening for infants' and toddlers' dietary quality through maternal diet. MCN Am J Matern Child Nurs 2005; 30(1):60-6.

9. Skinner JD, Carruth BR, Wendy B, Ziegler PJ. Children's food preferences: a longitudinal analysis. J Am Diet Assoc 2002; 102(11):1638-47.

10. Lipchock SV, Reed DR, Mennella JA. The gustatory and olfactory systems during infancy: implications for development of feeding behaviors in the high-risk neonate. Clin $\mathrm{Pe}$ rinatol 2011; 38(4):627-41.

11. Ventura AK, Worobey J. Early influences on the development of food preferences. Curr Biol 2013; 23(9):401-8.

12. Ministério da Saúde. Dez passos para alimentação saúdavel para crianças menores de dois anos. Brasília, DF: Ministério da Saúde; 2010.

13. Serra Majem L, Garcia Closas R, Ramon JM, Manau C, Cuenca E, Krasse B. Dietary habits and dental caries in a population of Spanish schoolchildren with low levels of caries experience. Caries Res 1993; 27(6):488-94.

14. Brekke HK, van Odijk J, Ludvigsson J. Predictors and dietary consequences of frequent intake of high-sugar, low-nutrient foods in 1-year-old children participating in the ABIS study. Br J Nutr 2007;97(1):176-81.

15. Cantoral A, Tellez-Rojo MM, Ettinger AS, Hu H, Hernandez-Avila M, Peterson K. Early introduction and cumulative consumption of sugar-sweetened beverages during the preschool period and risk of obesity at 8-14 years of age. Pediatr Obes 2016; 11(1):68-74.

16. Spahn JM, Reeves RS, Keim KS, Laquatra I, Kellogg M, Jortberg B, Clark NA. State of the evidence regarding behavior change theories and strategies in nutrition counseling to facilitate health and food behavior change. J Am Diet Assoc 2010; 110(6):879-91.

17. Ruottinen S, Niinikoski H, Lagstrom H, Rönnemaa T, Hakanen M, Viikari J, et al. High sucrose intake is associated with poor quality of diet and growth between 13 months and 9 years of age: the special Turku Coronary Risk Factor Intervention Project. Pediatrics 2008; 121(6):1676-85.

18. Organização Mundial da Saúde. WHO opens public consultation on draft sugars guideline. [accessed May 2018]. Disponível em URL: http://www.who.int/nutrition/sugars_public_consultation/en/. 2014.

19. Clark-Gambelunghe MB, Clark DA. Sensory development. Pediatr Clin North Am 2015; 62:367-84

20. Hamel C, Stevens A, Singh K, Ansari MT, Myers E, Ziegler $\mathrm{P}$, et al. Do sugar-sweetened beverages cause adverse health outcomes in adults? A systematic review protocol. Syst Rev 2014; 3(3):108-16

21. Sharlin JES. Essential of life cycle nutrition. Chapter 1: Nutrition Requirements During Pregnancy. Jones and Bartllet Plubishers; 2011.

22. Verloigne M, Van Lippevelde W, Maes L, Brug J, De Bourdeaudhuij I. Family- and school-based predictors of energy balance-related behaviours in children: a 6 -year longitudinal study. Public Health Nutr 2013; 16(8):202-11.

23. Lindsay AC, Sussner KM, Kim J, Gortmaker S. The role of parents in preventing childhood obesity. Future Child 2006; 16(1):169-86

24. Verzeletti C, Maes L, Santinello M, Vereecken CA. Soft drink consumption in adolescence: associations with food-related lifestyles and family rules in Belgium Flanders and the Veneto Region of Italy. Eur J Public Health 2010; 20(3):312-
25. Oliveria SA, Ellison RC, Moore LL, Gillman MW, Garrahie EJ, Singer MR. Parent-child relationships in nutrient intake: the Framingham Children's Study. Am J Clin Nutr 1992; 56(3):593-8.

26. Azevedo MS, Romano AR, Dos Santos Ida S, Cenci MS. Knowledge and beliefs concerning early childhood caries from mothers of children ages zero to 12 months. Pediatr Dent 2014; 36(3):95-9.

27. Hurd M. Anchoring and acquiescence bias in measuring assets in household surveys. J Risk Uncertainty 1999; 19(19):111-36

28. Barros AJ, Hirakata VN. Alternatives for logistic regression in cross-sectional studies: an empirical comparison of models that directly estimate the prevalence ratio. BMC Med Res Methodol 2003; 3(3):21-33

\section{Endereço para correspondência:}

Andréia Drawanz Hartwig

Programa de Pós-Graduação em Odontologia

Universidade Federal de Pelotas

Rua Gonçalves Chaves, 457

96015-560, Pelotas, RS, Brasil

Telefone: (53) 32226690 - ramal 135

E-mail: andreiahartwig@hotmail.com

Recebido: 24/06/18. Aceito: 16/07/18. 